Article

\title{
Early Diagnosis of Children with Classic Congenital Adrenal Hyperplasia Due to 21-Hydroxylase Deficiency by Newborn Screening
}

\section{Helmuth G. Dörr ${ }^{1, *}$, Birgit Odenwald ${ }^{2}$ and Uta Nennstiel-Ratzel ${ }^{2}$}

1 Division Pediatric Endocrinology, Department of Pediatrics, University of Hospital Erlangen, Loschgestr. 15, 91054 Erlangen, Germany

2 Bavarian Health and Food Safety Authority, 85764 Oberschleissheim, Germany; E-Mails: Birgit.Odenwald@lgl.bayern.de (B.O.); Uta.Nennstiel-Ratzel@lgl.bayern.de (U.N.-R.)

* Author to whom correspondence should be addressed;

E-Mail: helmuth-guenther.doerr@uk-erlangen.de; Tel.: +49-9131-853-3732.

Academic Editor: Ralph Fingerhut

Received: 4 February 2015 / Accepted: 11 June 2015 / Published: 17 June 2015

\begin{abstract}
Congenital adrenal hyperplasia $(\mathrm{CAH})$ comprises a group of rare autosomal recessively inherited disorders of cortisol biosynthesis in the adrenal cortex. More than $95 \%$ are based on a defect in the CYP21A2 gene causing 21-hydroxylase deficiency. Newborn screening (NBS) for CAH by means of 17-hydroxy-progesterone (17-OHP) determination in dried whole blood on filter paper has been introduced as part of the NBS in many countries worldwide. The goals of CAH screening are early detection of the severe, salt-wasting form, therefore prevention of adrenal crisis or death, early detection of the simple virilizing form, and prevention or shortening of the period of incorrect gender assignment in females. Methodological problems of false-positive samples, especially in pre-term infants, can be corrected by adapting the cut-off values for 17-OHP to birth weight, gestational age and age at the time of collection and by performing a second tier screening. Despite a positive voting for newborn CAH screening by the European Society for Pediatric Endocrinology and the Lawson Wilkins Pediatric Endocrine Society, it is obvious that the acceptance of CAH screening varies worldwide.
\end{abstract}

Keywords: CAH; newborn screening; 17OHP; CYP21A2; adrenal crisis 


\section{Introduction}

Congenital adrenal hyperplasia $(\mathrm{CAH})$ comprises a group of rare autosomal recessively inherited disorders of cortisol biosynthesis in the adrenal cortex. More than $95 \%$ are based on a defect in the CYP21A2 gene causing 21-hydroxylase deficiency. The classic defect occurs in two forms as CAH with salt wasting, which is three times more frequent, and as simple virilizing $\mathrm{CAH}$ without salt loss [1-3]. The worldwide incidence of classic $\mathrm{CAH}$ varies according to ethnic and geographic areas. The reported incidence is 1:8900 [4] in Argentina, between 1:15,000 and 1:16,000 in Europe and North America and 1:19,000 in Japan [5-7]. In contrast to the classic defect, the incidence of the non-classic defect (NCCAH) is assumed to be 1:1000 [8]. Due to intrauterine high androgen levels, the external genitalia of the female neonate with classic $\mathrm{CAH}$ is already virilized at birth, whereas the internal genitalia is always female. The degree of virilization (disorder of sex development; DSD, $46 \mathrm{XX}$ ) varies from slight enlargement of the clitoris (Prader stage 1) to complete masculinization of the external genitalia with a clitoris resembling a male phallus (Prader stage 5). In neonates and young infants, diffuse hyperplasia of the adrenal cortex can in most cases be imaged well with ultrasound. In boys and girls who are not diagnosed with the uncomplicated form of $\mathrm{CAH}$ without salt wasting at birth, pseudo-precocious puberty occurs from infancy due to an increased adrenal androgen production. In untreated children, there is premature closure of the epiphyses, and the untreated adult CAH patient is of short stature.

In children with the salt-wasting form of $\mathrm{CAH}$, the potentially fatal salt-losing crisis commonly begins between the second and third weeks of life with poor drinking, vomiting, dehydration, electrolyte changes (hyperpotassemia, hyponatremia), metabolic acidosis and increasing apathy. In children with $\mathrm{CAH}$, adrenal crisis occurring within the scope of stress situations is especially feared and cases of death are reported in the literature in this context. Deaths in $\mathrm{CAH}$ children were already reported in the literature in 1962 [9]. Mortality associated with CAH in children is reported to be between 3\% and 13\% [10-13].

The clinical diagnosis of classic CAH can be made at birth based on ambiguous genitalia in a newborn female (DSD 46, XX) or during the neonatal period in both sexes based on salt-wasting crisis. Elevated levels of 17-hydroxyprogesterone (17-OHP) in the serum/plasma confirm the diagnosis of classic 21-hydroxylase defect. Besides the 17-OHP concentrations, the serum levels of 21-deoxycortisol, androstenedione and testosterone are also high. A characteristic profile can be found in urine by means of capillary gas chromatography and mass spectrometry. Pregnanetriol, the specific urinary metabolite of 17-OHP, is massively increased. The quantitative determination of pregnanetriolone, which is practically undetectable under physiological conditions, is likewise a good parameter to confirm the diagnosis. In patients with salt-losing syndrome, the plasma renin activity (PRA) and the renin concentrations are elevated.

Treatment of choice is lifelong substitution with glucocorticoids and additionally with mineralocorticoids in cases with salt wasting. Until completion of growth, physiological hydrocortisone is the medication of choice. The dose requirement is determined individually. The daily dose of $10-15 \mathrm{mg} / \mathrm{m}^{2}$ body surface area is often subdivided into three single doses (morning dose about $50 \%$ of the daily dose). In salt-losing syndrome, the mineralocorticoid $9 \alpha$ fludrocortisone is administered in an age-dependent absolute dose of 50-200 $\mu \mathrm{g} /$ day. Most young infants with salt wasting require oral sodium supplements during the first months of life. Long-term treatment must not be interrupted or even discontinued. Detailed and repeated instructions of the parents and patients are required to enable them to increase in 
dose immediately and on their own as necessary in the event of high fever, gastroenteritis, beginning infections or occurrence of other acute stress situations The dose of hydrocortisone must be increased in all stress situations, without delay up to fivefold in accordance with healthy adrenal stress response. If oral medication is not possible for some reason (e.g., vomiting), hydrocortisone should be given in the form of an intramuscular injection or rectal suppositories as at-home emergency therapy or parenterally in the hospital. All patients with $\mathrm{CAH}$ must have an emergency identification document. After completion of growth, adolescents and young adults can be switched to other synthetic glucocorticoids. The transition to an adult endocrinologist should be well controlled.

\section{Newborn Screening for Classic CAH}

The goals of classic CAH newborn screening are early detection of the severe, salt-wasting form, therefore prevention of adrenal crisis or death, early detection of the simple virilizing form, and prevention or shortening of the period of incorrect gender assignment in females. The screening was not introduced to detect newborns with the non-classic form of $\mathrm{CAH}$.

Newborn screening (NBS) for CAH by means of 17-hydroxyprogesterone (17-OHP) determination in dried whole blood on filter paper was introduced already in 1977 by Pang et al. [14]. After various pilot projects [15-17], general NBS for classic CAH was introduced as part of the NBS in many countries worldwide [7,18-24]. However, in the beginning of newborn screening, many laboratories have used an immunologic method to measure 17-OHP. This method had a high number of false-positive results attributable to cross-reactivity with other steroids, and stress-induced high concentrations of 17-OHP in premature babies $[25,26]$. By adjustment of 17-OHP cutoff values for birth weight, gestational age, and sampling age $[27,28]$, a clear reduction of the false positive results could be achieved. However, approximately $1 \%$ of newborns still must be retested. To further improve CAH neonatal screening, new strategies have been developed and introduced in the NBS such as a second-tier CAH assay in which the conventional immunologic method was followed by Tandem mass spectrometry (MS/MS) which offers a high level of analytical sensitivity [29,30].

The benefit of NBS for classic CAH was emphasized in a common consensus paper published by the European Society for Pediatric Endocrinology and the Lawson Wilkins Pediatric Endocrine Society [31]. However, there are still pediatric endocrinologists who doubt the benefit of newborn CAH screening, arguing that, in modern healthcare systems, mortality from salt wasting is very unlikely, false gender assignment could not always be prevented (see case report below) and the costs of NBS are high owing to second tier steps for reducing the high rate of recall in preterm infants [32,33]. Moreover, it has been shown in France that NBS allowed clinical diagnosis of CAH in 162 of 383 cases $(42.3 \%)$, while the other cases were detected clinically or through family history [34]. Thus, it is not surprising that $\mathrm{CAH}$ screening has met with varying acceptance worldwide. To our knowledge, CAH screening is currently not performed in various European countries such as Greece, Ireland, Portugal and the UK [33], and in Australia [35]. 


\section{Problems with False-Positive and False Negative-Results}

Newborn screening based on measuring 17-OHP by immunoassay generated a high number of false-positive results in the past [21,36]. Even the collection of samples within $24 \mathrm{~h}$ of life can increase the rate of false positivity. Low birth weight, prematurity and birth stress cause high 17-OHP levels [25,27,37]. Gidlof et al. analysed the screening data from Sweden where 2737932 infants (99.8\%) underwent testing between 1 January 1986 until 31 December 2011 [23]. They identified 1497 infants with false-positive findings. The positive predictive value was higher in full-term (25.1\%) than preterm $(1.4 \%)$ infants and correlated with gestational age $(\mathrm{r}=0.98 ; p<0.001)$. The recall rate in full-term infants $(0.03 \%)$ was lower $(p<0.001)$ than that in preterm infants $(0.57 \%)$. However, by using $17 \mathrm{OHP}$ cutoff values adjusted by birthweight and/or gestational age, it was possible to reduce the recall rate from $1.12 \%$ to $0.5 \%$ in Germany [28]. Other newborn CAH screening protocols with second tier steps such as re-measuring first-tier elevated 17-OHP levels by liquid chromatography-tandem mass spectrometry [38], by steroid profiling of steroids such as 17-OHP, androstenedione, and cortisol [39-42] and/or by molecular-genetic analyses [43] improve screening effectiveness. According to the results of Kosel et al., further reduction of the recall rates of around $90 \%$ without loss of sensitivity was possible by analyzing the CYP21A2-gene as second-tier [44].

Besides the problem of false-positive results, there is also the problem of false-negative results. It is very difficult to grasp false negative cases. It has been shown that 17-OHP levels increase with time in children affected with CAH. Thus, the accuracy of the diagnosis is relatively poor during the first two postnatal days [45]. Newborns affected with CAH having a certain genotype can have normal 17-OHP levels in NBS [46]. Newborns with CAH, who were treated systemically with glucocorticoids during the postnatal period, have low 17-OHP levels [47].

Recently we evaluated the CAH screening quality and outcome in Bavaria from 1999 to 2011 and found among 1,624,146 screened infants 114 cases of classic CAH with a prevalence of 1:14,247 [48]. In our report, we identified five infants who had misleading normal results at first screen, connected with mild CYPA2 mutations, in particular in newborns with simple virilizing $\mathrm{CAH}$ and the mutation Ile172Asn, or caused by neonatal steroid medication.

\section{Case Report}

A full-term baby (normal birth weight and size) with abnormal external genitalia was born in a small hospital. The midwife and the pediatrician stated: boy with hypospadia. The child was named "Paul" by the parents and was registered as a boy. Newborn screening was performed on the 3rd day. On the 5th day, a pathologically high 17-OHP level of $340 \mathrm{nmol} / \mathrm{L}$ was detected, and the screening laboratory suspected CAH with 21- hydroxylase deficiency. At the age of 8 days, the newborn was referred to our hospital for further diagnostic investigation. During examination, we found ambiguous genitalia with no palpable testes (Prader stage 4). When admitted to hospital, the "boy" emptied blood through the penile orifice. Chemical analysis showed a low serum sodium concentration of $128 \mathrm{mmol} / \mathrm{L}$ and a high potassium concentration of $6.3 \mathrm{mmol} / \mathrm{L}$. The diagnosis of CAH with 21 - hydroxylase deficiency defect was confirmed (serum 17-OHP $420 \mathrm{nmol} / \mathrm{L}$ ), and substitution therapy with hydrocortisone and fludrocortisone was initiated. The "boy" became a girl and was renamed as "Paula". 
This case demonstrates that screening allowed early diagnosis and early therapy, but did not allow correct sex assignment.

Persons involved in delivery, such as midwives, nurses or physicians, should not make any statements regarding the sex of the newborn if genital findings are not clear.

\section{Non-Classic CAH (NCCAH)}

In some cases, diagnosis of children with non-classic CAH by NBS was reported in the literature [23]. The evaluation of the screening results of 4.5 million Japanese newborns between 1991 and 1994 only yielded evidence of 4 cases with non-classic $\mathrm{CAH}$ [49]. A mutation on an allele leading to the complete loss of enzyme activity was detected in the 4 patients. Children with NCCAH do not warrant early detection through neonatal screening.

\section{Conclusions}

It is one of the specific features of $\mathrm{CAH}$ newborn screening that classic $\mathrm{CAH}$ can only be suspected based on monitoring and increasing 17-OHP levels. CAH screening is not considered completed until reliable documentation of normal levels is available. However, one has to keep in mind that a small group of newborns with uncomplicated $\mathrm{CAH}$ without salt loss and certain gene mutations might be identified clinically only in the future. A newborn with extremely high 17OHP-levels should be presented immediately to a pediatric endocrinologist. The clinical course in patients with classic $\mathrm{CAH}$ and 21-hydroxylase defect depends on early diagnosis of the disease. Positive findings of screening for $\mathrm{CAH}$ need to be verified quickly by clinical and endocrinological evidence. The patients identified by screening should be treated by pediatricians within the scope of medical family care in close cooperation with pediatric endocrinologists.

\section{Key Notes}

- Classic congenital adrenal hyperplasia (CAH) with 21- hydroxylase deficiency (CYP21A2) is a rare autosomal recessive hereditary disorder of adrenal steroid biosynthesis.

- Incidence varies in different ethnic groups; in classic CAH, it is approximately $1: 14,000$ to 1: 18,000 births.

- Newborn screening for classic CAH enables early therapy to prevent salt-losing crises and, as a rule, correct sex assignment and normal growth.

- Samples for newborn screening should be collected on the 3rd day of life.

- Methodological problems of false-positive samples, especially in pre-term infants, can be corrected by adapting the cut-off values for 17-OHP to birth weight, gestational age and age at the time of collection and by performing a second tier screening.

- In extremely high 17-OHP levels, the newborns should immediately be presented to the pediatric endocrinologist since salt-losing crisis may occur early. 


\section{Author Contributions}

All authors have participated in the concept and design, analysis and interpretation of data, drafting or revising of the manuscript; and they have approved the manuscript as submitted.

\section{Conflicts of Interest}

The authors declare no conflict of interest.

\section{References}

1. Miller, W.L.; Auchus, R.J. The molecular biology, biochemistry, and physiology of human steroidogenesis and its disorders. Endocr. Rev. 2011, 32, 81-151.

2. New, M.I. An update of congenital adrenal hyperplasia. Ann. N. Y. Acad. Sci. 2004, 1038, 14-43.

3. Speiser, P.W.; White, P.C. Congenital adrenal hyperplasia. N. Engl. J. Med. 2003, 349, 776-788.

4. Grunieiro-Papendieck, L.; Chiesa, A.; Mendez, V.; Prieto, L. Neonatal screening for congenital adrenal hyperplasia: Experience and results in Argentina. J. Pediatr. Endocrinol. Metab. 2008, 21, 73-78.

5. Van der Kamp, H.J.; Wit, J.M. Neonatal screening for congenital adrenal hyperplasia. Eur. J. Endocrinol. 2004, 151 (Suppl. 3), U71-U75.

6. White, P.C. Neonatal screening for congenital adrenal hyperplasia. Nat. Rev. Endocrinol. 2009, 5, 490-498.

7. Tajima, T.; Fujikura, K.; Fukushi, M.; Hotsubo, T.; Mitsuhashi, Y. Neonatal screening for congenital adrenal hyperplasia in Japan. Pediatr. Endocrinol. Rev. 2012, 10 (Suppl. 1), 72-78.

8. Witchel, S.F. Non-classic congenital adrenal hyperplasia. Steroids 2013, 78, 747-750.

9. Cleveland, W.W.; Green, O.C.; Wilkins, L. Deaths in congenital adrenal hyperplasia. Pediatrics 1962, 29, 3-17.

10. Jaaskelainen, J.; Voutilainen, R. Long-term outcome of classical 21-hydroxylase deficiency: Diagnosis, complications and quality of life. Acta Paediatr. 2000, 89, 183-187.

11. Lim, Y.J.; Batch, J.A.; Warne, G.L. Adrenal 21-hydroxylase deficiency in childhood: 25 years' experience. J. Paediatr. Child Health 1995, 31, 222-227.

12. Thilen, A.; Larsson, A. Congenital adrenal hyperplasia in Sweden 1969-1986. Prevalence, symptoms and age at diagnosis. Acta Paediatr. Scand. 1990, 79, 168-175.

13. Swerdlow, A.J.; Higgins, C.D.; Brook, C.G.; Dunger, D.B.; Hindmarsh, P.C.; Price, D.A.; Savage, M.O. Mortality in patients with congenital adrenal hyperplasia: A cohort study. J. Pediatr. 1998, 133, 516-520.

14. Pang, S.; Hotchkiss, J.; Drash, A.L.; Levine, L.S.; New, M.I. Microfilter paper method for 17 alpha-hydroxyprogesterone radioimmunoassay: Its application for rapid screening for congenital adrenal hyperplasia. J. Clin. Endocrinol. Metab. 1977, 45, 1003-1008.

15. Valentino, R.; Tommaselli, A.P.; Rossi, R.; Lombardi, G.; Varrone, S. A pilot study for neonatal screening of congenital adrenal hyperplasia due to 21-hydroxylase and 11-beta-hydroxylase deficiency in Campania region. J. Endocrinol. Investig. 1990, 13, 221-225. 
16. Chu, S.Y.; Tsai, W.Y.; Chen, L.H.; Wei, M.L.; Chien, Y.H.; Hwu, W.L. Neonatal screening for congenital adrenal hyperplasia in Taiwan: A pilot study. J. Formos. Med. Assoc. 2002, 101, 691-694.

17. Gleeson, H.K.; Wiley, V.; Wilcken, B.; Elliott, E.; Cowell, C.; Thonsett, M.; Byrne, G.; Ambler, G. Two-year pilot study of newborn screening for congenital adrenal hyperplasia in New South Wales compared with nationwide case surveillance in Australia. J. Paediatr. Child Health 2008, 44, 554-559.

18. Pang, S.; Clark, A. Newborn screening, prenatal diagnosis, and prenatal treatment of congenital adrenal hyperplasia due to 21-hydroxylase deficiency. Trends Endocrinol. Metab. 1990, 1, 300-307.

19. Pang, S. Newborn screening for congenital adrenal hyperplasia. Pediatr. Ann. 2003, 32, 516-523.

20. Pang, S.Y.; Wallace, M.A.; Hofman, L.; Thuline, H.C.; Dorche, C.; Lyon, I.C.; Dobbins, R.H.; Kling, S.; Fujieda, K.; Suwa, S. Worldwide experience in newborn screening for classical congenital adrenal hyperplasia due to 21-hydroxylase deficiency. Pediatrics 1988, 81, 866-874.

21. Van der Kamp, H.J.; Noordam, K.; Elvers, B.; Van Baarle, M.; Otten, B.J.; Verkerk, P.H. Newborn screening for congenital adrenal hyperplasia in The Netherlands. Pediatrics 2001, 108, 1320-1324.

22. Nascimento, M.L.; Cristiano, A.N.; Campos, T.; Ohira, M.; Cechinel, E.; Simoni, G.; Lee, J.; Linhares, R.M.; Silva, P.C. Ten-year evaluation of a neonatal screening program for congenital adrenal hyperplasia. Arq. Bras. Endocrinol. Metab. 2014, 58, 765-771.

23. Gidlof, S.; Wedell, A.; Guthenberg, C.; von Dobeln, U.; Nordenstrom, A. Nationwide neonatal screening for congenital adrenal hyperplasia in Sweden: A 26-year longitudinal prospective population-based study. JAMA Pediatr. 2014, 168, 567-574.

24. Gonzalez, E.C.; Carvajal, F.; Frometa, A.; Arteaga, A.L.; Castells, E.M.; Espinosa, T.; Coto, R.; Perez, P.L.; Tejeda, Y.; Del Rio, L.; et al. Newborn screening for congenital adrenal hyperplasia in Cuba: Six years of experience. Clin. Chim. Acta 2013, 421, 73-78.

25. Al-Nuaim, A.R.; Abdullah, M.A.; Stevens, B.; Zain, M. Effect of gender, birth weight and gestational age on serum 17-hydroxyprogesterone concentration and distribution among neonates in Saudi Arabia. Indian J. Pediatr. 1995, 62, 605-609.

26. Balsamo, A.; Cacciari, E.; Piazzi, S.; Cassio, A.; Bozza, D.; Pirazzoli, P.; Zappulla, F. Congenital adrenal hyperplasia: Neonatal mass screening compared with clinical diagnosis only in the Emilia-Romagna region of Italy, 1980-1995. Pediatrics 1996, 98, 362-367.

27. Allen, D.B.; Hoffman, G.L.; Fitzpatrick, P.; Laessig, R.; Maby, S.; Slyper, A. Improved precision of newborn screening for congenital adrenal hyperplasia using weight-adjusted criteria for 17-hydroxyprogesterone levels. J. Pediatr. 1997, 130, 128-133.

28. Olgemoller, B.; Roscher, A.A.; Liebl, B.; Fingerhut, R. Screening for congenital adrenal hyperplasia: Adjustment of 17-hydroxyprogesterone cut-off values to both age and birth weight markedly improves the predictive value. J. Clin. Endocrinol. Metab. 2003, 88, 5790-5794.

29. De Jesus, V.R.; Simms, D.A.; Schiffer, J.; Kennedy, M.; Mei, J.V.; Hannon, W.H. Pilot proficiency testing study for second tier congenital adrenal hyperplasia newborn screening. Clin. Chim. Acta 2010, 411, 1684-1687.

30. Schwarz, E.; Liu, A.; Randall, H.; Haslip, C.; Keune, F.; Murray, M.; Longo, N.; Pasquali, M. Use of steroid profiling by UPLC-MS/MS as a second tier test in newborn screening for congenital adrenal hyperplasia: The Utah experience. Pediatr. Res. 2009, 66, 230-235. 
31. Consensus statement on 21-hydroxylase deficiency from the Lawson Wilkins pediatric endocrine society and the European society for paediatric endocrinology. J. Clin. Endocrinol. Metab. 2002, 87, 4048-4053.

32. Grosse, S.D.; Van Vliet, G. How many deaths can be prevented by newborn screening for congenital adrenal hyperplasia? Horm. Res. 2007, 67, 284-291.

33. Hird, B.E.; Tetlow, L.; Tobi, S.; Patel, L.; Clayton, P.E. No evidence of an increase in early infant mortality from congenital adrenal hyperplasia in the absence of screening. Arch. Dis. Child. 2014, 99, $158-164$.

34. Coulm, B.; Coste, J.; Tardy, V.; Ecosse, E.; Roussey, M.; Morel, Y.; Carel, J.C. Efficiency of neonatal screening for congenital adrenal hyperplasia due to 21-hydroxylase deficiency in children born in mainland France between 1996 and 2003. Arch. Pediatr. Adolesc. Med. 2012, 166, 113-120.

35. Wu, J.Y.; Sudeep; Cowley, D.M.; Harris, M.; McGown, I.N.; Cotterill, A.M. Is it time to commence newborn screening for congenital adrenal hyperplasia in Australia? Med. J. Aust. 2011, 195, 260-262.

36. Gurian, E.A.; Kinnamon, D.D.; Henry, J.J.; Waisbren, S.E. Expanded newborn screening for biochemical disorders: The effect of a false-positive result. Pediatrics 2006, 117, 1915-1921.

37. Gruneiro-Papendieck, L.; Prieto, L.; Chiesa, A.; Bengolea, S.; Bossi, G.; Bergada, C. Neonatal screening program for congenital adrenal hyperplasia: Adjustments to the recall protocol. Horm. Res. 2001, 55, 271-277.

38. Minutti, C.Z.; Lacey, J.M.; Magera, M.J.; Hahn, S.H.; McCann, M.; Schulze, A.; Cheillan, D.; Dorche, C.; Chace, D.H.; Lymp, J.F.; et al. Steroid profiling by tandem mass spectrometry improves the positive predictive value of newborn screening for congenital adrenal hyperplasia. J. Clin. Endocrinol. Metab. 2004, 89, 3687-3693.

39. Sarafoglou, K.; Banks, K.; Gaviglio, A.; Hietala, A.; McCann, M.; Thomas, W. Comparison of one-tier and two-tier newborn screening metrics for congenital adrenal hyperplasia. Pediatrics 2012, 130, e1261-e1268.

40. Seo, J.Y.; Park, H.D.; Kim, J.W.; Oh, H.J.; Yang, J.S.; Chang, Y.S.; Park, W.S.; Lee, S.Y. Steroid profiling for congenital adrenal hyperplasia by tandem mass spectrometry as a second-tier test reduces follow-up burdens in a tertiary care hospital: A retrospective and prospective evaluation. J. Perinat. Med. 2014, 42, 121-127.

41. Sarafoglou, K.; Gaviglio, A.; Hietala, A.; Frogner, G.; Banks, K.; McCann, M.; Thomas, W. Comparison of newborn screening protocols for congenital adrenal hyperplasia in preterm infants. J. Pediatr. 2014, 164, 1136-1140.

42. Janzen, N.; Sander, S.; Terhardt, M.; Steuerwald, U.; Peter, M.; Das, A.M.; Sander, J. Rapid steroid hormone quantification for congenital adrenal hyperplasia (CAH) in dried blood spots using UPLC liquid chromatography-tandem mass spectrometry. Steroids 2011, 76, 1437-1442.

43. Krone, N.; Braun, A.; Weinert, S.; Peter, M.; Roscher, A.A.; Partsch, C.J.; Sippell, W.G. Multiplex minisequencing of the 21-hydroxylase gene as a rapid strategy to confirm congenital adrenal hyperplasia. Clin. Chem. 2002, 48, 818-825.

44. Kosel, S.; Burggraf, S.; Fingerhut, R.; Dorr, H.G.; Roscher, A.A.; Olgemoller, B. Rapid second-tier molecular genetic analysis for congenital adrenal hyperplasia attributable to steroid 21hydroxylase deficiency. Clin. Chem. 2005, 51, 298-304. 
45. White, P.C. Optimizing newborn screening for congenital adrenal hyperplasia. J. Pediatr. 2013, 163, $10-12$.

46. Schreiner, F.; Brack, C.; Salzgeber, K.; Vorhoff, W.; Woelfle, J.; Gohlke, B. False negative 17-hydroxyprogesterone screening in children with classical congenital adrenal hyperplasia. Eur. J. Pediatr. 2008, 167, 479-481.

47. Rohrer, T.R.; Gassmann, K.F.; Pavel, M.E.; Dorr, H.G. Pitfall of newborn screening for congenital adrenal hyperplasia due to 21-hydroxylase deficiency. Biol. Neonate 2003, 83, 65-68.

48. Odenwald, B.; Doerr, H.; Bonfig, W.; Schmidt, H.; Wildner, M.; Nennstiel-Ratzel, U. Classic congenital adrenal hyperplasia due to 21-hydroxylase-deficiency: 13 years of neonatal screening and follow-up in Bavaria. Klinische Pädiatr. 2015, in press.

49. Tajima, T.; Fujieda, K.; Nakae, J.; Toyoura, T.; Shimozawa, K.; Kusuda, S.; Goji, K.; Nagashima, T.; Cutler, G.B., Jr. Molecular basis of nonclassical steroid 21-hydroxylase deficiency detected by neonatal mass screening in Japan. J. Clin. Endocrinol. Metab. 1997, 82, 2350-2356.

(C) 2015 by the authors; licensee MDPI, Basel, Switzerland. This article is an open access article distributed under the terms and conditions of the Creative Commons Attribution license (http://creativecommons.org/licenses/by/4.0/). 